\title{
THE ETIOLOGY AND MAINTENANCE OF SEVERE ANIMAL PHOBIAS
}

\author{
Richard J. McNally* and Gail S. Steketee \\ Department of Psychiatry, Temple University School of Medicine, Philadelphia, Penn., U.S.A.
}

(Received 21 November 1984)

\begin{abstract}
Summary-Twenty-two outpatients who sought behavioral treatment for a severe animal phobia were questioned via structured interview to obtain information on the mode of onset and course of development of their fear. Information was also obtained regarding feared consequences and distressing stimulus characteristics of their feared animal. In each case, the phobia began in early childhood and remained stable or worsened with age. Mode of onset was unclassifiable in 15 cases $(68 \%)$ because of patient inability to remember precipitants. Of the remaining 7 patients, 5 ascribed their fear to a frightening encounter with the animal ('conditioning'); indirect ('instructional' and 'vicarious') causes were cited in the remaining cases. Virtually all patients feared panic and its consequences following an unavoidable encounter with the phobic animal, whereas less than half reported fear that the animal would attack. Stimulus features, especially movement, were found to be salient fear-eliciting cues.
\end{abstract}

\section{INTRODUCTION}

Behavior therapists have traditionally viewed phobic fears as learned responses established through classical conditioning (e.g. Wolpe and Rachman, 1960). This view influenced the development of treatment techniques designed to decondition fear such as systematic desensitization (Wolpe, 1958) and implosive therapy (Stampfl and Levis, 1967). Although one cannot infer etiology from treatment response, the success of these techniques probably strengthened the belief that phobias are, indeed, conditioned phenomena.

Despite its heuristic value, several authors have questioned the adequacy of the classical conditioning theory of phobias. Some have suggested that the traditional model may be salvaged with the addition of concepts such as fear incubation (Eysenck, 1979) or preparedness (Seligman, 1971). Others (Reiss, 1980; Reiss and McNally, 1985) have advised that conditioning theories of phobia acquisition should be based on contemporary informational models (e.g. Rescorla, 1978) rather than on the obsolete contiguity model. Still others have suggested that phobias may be learned in ways other than classical conditioning. Rachman (1977), for example, has suggested that phobias may not only be acquired via conditioning, but also through verbal information transmission and vicarious learning.

Rachman's (1977) postulation of 'three pathways to fear' has spurred several investigations into the etiology of phobias. Studying the origins of snake fear in college students, Murray and Foote (1979) found that most fearful students acquired their fear through verbal or vicarious means. Few $S$ s reported experiences that might be construed as conditioning events. In fact, the 3 individuals who had actually been bitten by snakes reported no snake fear.

Öst and Hugdahl (1981) classified the mode of fear acquisition in a sample of claustrophobics, social phobics and animal phobics. In contrast to Murray and Foote (1979), they found that 'conditioning experiences' accounted for fear onset twice as often as did verbal or vicarious experiences. In a subsequent investigation, Öst and Hugdahl (1983) reported that $87 \%$ of a series of agoraphobics attributed their fears to conditioning experiences. None of these patients implicated verbal factors, and only $9 \%$ ascribed the onset of their fears to vicarious learning.

The findings reported by Öst and Hugdahl are in striking contrast to the reports of others who maintain that conditioning plays a minimal role in the etiology of phobias, especially agoraphobia (e.g. Emmelkamp, 1982, pp. 17-22; Goldstein and Chambless, 1978). These inconsistencies may in part be due to varying definitions of what qualifies as a conditioning event (McNally, 1983).

*Present address: Department of Psychology, University of Health Sciences, The Chicago Medical School, Bldg 51, North Chicago, IL 60064, U.S.A. 
Theorists who view conditioning as a form of stimulus-stimulus (S-S) learning note that patients rarely report encountering painful 'unconditioned stimuli' (US) analogous to the electric shocks used in laboratory conditioning research. Accordingly, they question the adequacy of conditioning explanations (e.g. Emmelkamp, 1982, p. 19). On the other hand, theorists who conceptualize conditioning as stimulus-response (S-R) learning imply that events in which a person becomes extremely frightened qualify as conditioning, despite the absence of a specifiable US (e.g. Wolpe, 1982, p. 10).

The present paper reports an investigation into the etiology and maintenance of severe animal phobias. Twenty-two adults who sought behavior therapy for these fears were questioned via a structured interview to obtain information on the mode of onset and course of development of their phobia. Patients were also asked about the consequences they feared following an unavoidable encounter with the phobic animal because such expectations may play a role in mediating phobic behavior. Since several authors (Bennett-Levy and Marteau, 1984; Cornelius and Averill, 1983) have noted that animal fears may be based on aversion to certain attributes of the animal (e.g. the way it moves or sounds) rather than on any expectation of harm, each patient was asked which stimulus aspects of the phobic animal were particularly distressing.

\section{METHOD}

\section{Subjects}

The $S$ s were 22 adults (20 females and 2 males) who applied for behavioral treatment of a severe animal phobia at the Behavior Therapy Unit, Temple University School of Medicine. All met the DSM-III criteria for simple phobia (APA, 1980). There were 10 snake phobics, 4 cat phobics, 4 bird phobics, 2 dog phobics and 2 spider phobics. All were treated within the context of an outcome study which will be reported elsewhere.

Patients ranged in age from 22 to $57 \mathrm{yr}$ old $(\bar{X}=40.0 \mathrm{yr}, \mathrm{SD}=10.6 \mathrm{yr})$. Seventy-seven per cent were white and $23 \%$ were black.

All $S$ s were severely phobic. Only individuals who refused to reach into a cage containing the phobic animal during a behavioral avoidance test were accepted for the study. On the average, $S \mathrm{~s}$ were unable to proceed closer than $2 \mathrm{ft}$ from the closed cage (range $=0-10 \mathrm{ft}$ ). All $S$ s completed a 30-item true-false animal fear questionnaire, either the SNAQ (Lang, Melamed and Hart, 1970) or a version adapted to their particular fear. The mean pretreatment score was $24.4(\mathrm{SD}=7.3)$, which compares favorably with the mean of Lang et al.'s college student snake phobics $(\bar{X}=20.6)$. The present sample scored in the normal range $(\bar{X}=8.4, \mathrm{SD}=10.3)$ on the Beck Depression Inventory (BDI; Beck, 1967) with all but $3 S$ s scoring 12 or below.

\section{Procedure}

A structured interview was conducted with each patient (usually by telephone) to obtain information regarding the mode of onset, course of development and frequency of natural exposure to the phobic animal. Information was also obtained regarding the feared consequences that $S \mathrm{~s}$ expected to occur following an unavoidable encounter with their feared animal. $S$ s were also questioned about the specific stimulus characteristics of their feared animal that they found particularly distressing. A copy of the interview protocol is available from the authors.

\section{RESULTS}

Table 1 summarizes data on the mode of phobic onset, course of fear development, feared consequences and distressing stimulus characteristics of the feared animal.

\section{Mode of onset}

Most $S \mathrm{~s}(77 \%)$ could not recall the onset of their phobia, reporting that they had had the fear as long as they could remember. Two of these, however, stated that their parents had witnessed its origin. One case was classified as a 'conditioning' onset and the other as an 'instructional' onset. Thus, $68 \%$ of the cases were unclassifiable. 
Table 1. Variables associated with the etiology and maintenance of animal phobias

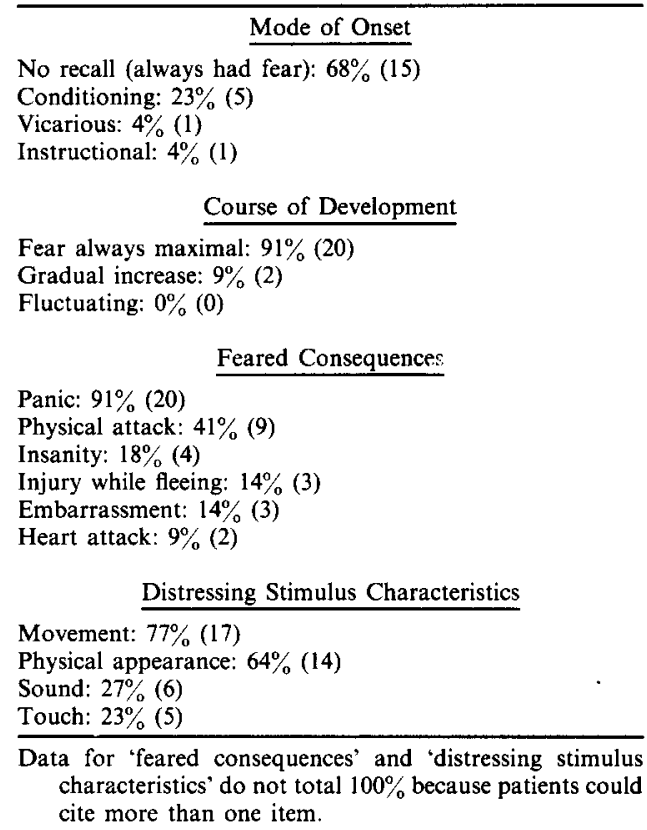

Five $S \mathrm{~s}(23 \%)$ attributed their fear to a frightening encounter with the animal and thus were classified as conditioning cases. In no instance, however, did the animal inflict pain. Thus these cases may be interpreted as instances of S-R conditioning.

Only one case involved an instructional onset. According to her mother, the patient acquired a bird phobia after her father teasingly said that birds might 'swoop down and get her'.

Although there were no cases of vicarious learning, per se, one patient attributed her snake phobia to watching frightening movies which depicted snakes as dangerous.

In summary, 7 cases out of 22 were classifiable in terms of onset. Of these, $71 \%$ were deemed instances of conditioning, whereas vicarious and instructional factors prevailed in the remaining cases.

\section{Course of development}

As noted earlier, most $S$ s could not recall the origin of their phobia. Of those who could, all said it began before the age of $10 \mathrm{yr}$. Nearly all patients $(91 \%)$ reported that once the fear was established, its intensity remained constant. The remaining $2 S$ s reported that phobic intensity increased gradually. None reported fluctuations in fear level.

Patients found it very difficult to estimate the amount of naturally-occurring exposure they had had to their feared animal following onset of their phobia. All, however, said they avoided contact as much as possible.

\section{Feared consequences}

Twenty patients $(91 \%)$ expected to panic in an unavoidable encounter with their feared animal. Nine patients $(41 \%)$ also believed that the animal would attack them (e.g. dog would bite). All other expected consequences were indirect effects of panic: going insane from fear $(18 \%)$, embarrassment at others' noticing one's fear $(14 \%)$, getting accidentally injured while trying to flee $(14 \%)$ and having a heart attack $(9 \%)$.

\section{Distressing stimulus characteristics}

Seventeen $S \mathrm{~s}(77 \%)$ stated that they found the way the animal moved to be very upsetting (e.g. slithering of a snake). Physical appearance, independent of movement, was cited second most frequently $(64 \%)$, followed by sounds made by the animal $(27 \%)$ and its tactile properties $(23 \%)$. 


\section{DISCUSSION}

Consistent with previous findings, all patients in this sample acquired their animal phobia during childhood (Marks and Gelder, 1966). The majority could not remember its onset, but of those who did, $71 \%$ attributed it to experiences interpretable as conditioning events involving frightening (but never painful) encounters with the animal. (This figure should be viewed with caution because it is based on only an $N=7$.) Our results are consistent with those of Öst and Hugdahl $(1981,1983)$ who found that $53 \%$ of their animal phobics acquired their fear through conditioning. Our findings, however, differ from those of Murray and Foote (1979) and Kleinknecht (1982) who reported that vicarious and instructional factors prevailed in the etiology of subclinical animal fears. Taken together, these findings suggest that very frightening experiences are more likely to be associated with the onset of clinical phobias, whereas milder fears may result from less direct forms of learning (e.g. verbal transmission of threatening information). Thus, different pathways to fear (Rachman, 1977) may be associated with fears of different severity.

Following fear onset, the course of development was remarkably stable. This is not representative of all individuals who, at some point in their lives, fear animals. Research has shown that the prevalence of animal phobia declines as a function of age group (Agras, Chapin and Oliveau, 1972). This suggests that although there are many children with animal fears, most lose them as they grow older. Thus, individuals presenting for treatment are typically adults whose childhood fear has failed to dissipate. It is not clear what variables differentiate children who lose their fear of animals from those who do not, although differential avoidance behavior is a possible explanation. All $S \mathrm{~s}$ consistently avoided their phobic animal and fled following surprise encounters, thus limiting possibilities for naturally-occurring fear reduction. Whether a person avoids may in turn depend on other variables such as the degree of anxiety tolerance (Reiss and McNally, 1985).

The most frequently cited feared consequence of encountering the phobic animal was the experience of panic. Although fear of panic is a salient characteristic of agoraphobia--for a review, see Foa, Steketee and Young (1984) - the present study corroborates recent research suggesting that it is not uncommon in other anxiety disorders (Reiss, Peterson, Gursky and McNally, 1984). Of course, the expectation of panic noted in the present sample is clearly related to specific environmental stimuli, in contrast to the often unpredictable panic attacks associated with the development of agoraphobia. In addition, agoraphobics exhibit less tolerance for anxiety than simple phobics (Reiss et al., 1984). Nonetheless, the present study indicates that an exaggerated estimate of the probability of physical harm is not necessarily the primary variable implicated in animal phobias.

With respect to fearsome characteristics of the animals, movement and physical appearance were cited most often, some patients also reported being disturbed by auditory and tactile aspects. Previous research has suggested that such perceptual features of the phobic animal are critical in controlling fear behavior (Bennett-Levy and Marteau, 1984; Cornelius and Averill, 1983). If so, these cues may need to be deliberately included in exposure treatments in order to maximize gains.

There are limitations in the present study, as in any retrospective analysis based on self-report. For most patients, no definite statement can be made regarding the mode of onset because of their lack of recall. These memories may, in part, be inaccessible because they were stored prior to the development of language. Moreover, even in those instances when patients did cite a specific event, it may not necessarily have been the actual cause of the phobia. It is possible that individuals postulate plausible triggering events when at a loss to explain the cause of their irrational fears. Therefore, more precisely, the present study concerns the casual attributions (which may or may not be accurate) made by patients to account for their phobia.

Acknowledgements - We would like to thank Edna B. Foa and Michael J. Kozak for their helpful comments on the interview protocol. Preparation of this manuscript was supported in part by National Institute of Mental Health Grant MH31634 awarded to Edna B. Foa.

Requests for reprints should be addressed to the first author.

\section{REFERENCES}

Agras W. S., Chapin H. N. and Oliveau D. C. (1972) The natural history of phobia: course and prognosis. Arch gen. Psychiat. 26, 315-317. 
APA; American Psychiatric Association (1980) Diagnostic and Statistical Manual of Mental Disorders, 3rd edn. APA, Washington, D.C.

Beck A. T. (1967) Depression: Causes and Treatment. Univ. of Pennsylvania Press, Philadelphia, Penn.

Bennett-Levy J. and Marteau T. (1984) Fear of animals: what is prepared? Br. J. Psychol. 75, 37-42.

Cornelius R. R. and Averill J. R. (1983) Sex differences in fear of spiders. J. Person. soc. Psychol. 45, 377-383.

Emmelkamp P. M. G. (1982) Phobic and Obsessive-Compulsive Disorders: Theory, Research and Practice. Plenum Press, New York.

Eysenck H. J. (1979) The conditioning model of neurosis. Behavl Brain Sci. 2, 155-166.

Foa E. B., Steketee G. and Young M. C. (1984) Agoraphobia: phenomenological aspects, associated characteristics and theoretical considerations. Clin. Psychol. Rev, 4, 431-457.

Goldstein A. J. and Chambless D. L. (1978) A reanalysis of agoraphobia. Behav. Ther. 9, 47-59.

Kleinknecht R. A. (1982) The origins and remission of fear in a group of tarantula enthusiasts. Behav. Res. Ther. 20 , 437-443.

Lang P. J., Melamed B. G. and Hart J. (1970) A psychophysiological analysis of fear modification using an automated desensitization procedure. J. abnorm. Psychol. 76, 220-234.

Marks I. M. and Gelder M. (1966) Different ages of onset in varieties of phobia. Am. J. Psychiat. 123, $218-221$.

McNally R. J. (1983) Phobic and obsessive-compulsive disorders: current status of theory and treatment. Contemp. Psychol. 28, 776-778.

Murray E. J. and Foote F. (1979) The origins of fear of snakes. Behav. Res. Ther. 17, 489-493.

Ost L.-G. and Hugdahl K. (1981) Acquisition of phobias and anxiety response patterns in clinical patients. Behav. Res. Ther. 19, 439-447.

Öst L.-G. and Hugdahl K. (1983) Acquisition of agoraphobia, mode of onset and anxiety response patterns. Behav. Res. Ther. 21, 623-631.

Rachman S. (1977) The conditioning theory of fear-acquisition: a critical examination. Behav. Res. Ther. 15, 375-387.

Reiss S. (1980) Pavlovian conditioning and human fear: an expectancy model. Behav. Ther. 11, 380-396.

Reiss S. and McNally R. J. (1985) The expectancy model of fear. In Theoretical Issues in Behavior Therapy (Edited by Reiss S. and Bootzin R. R.), pp. 107-121. Academic Press, New York.

Reiss S., Peterson R. A., Gursky D. M. and McNally R. J. (1984) Anxiety sensitivity: a new personality variable relevant to psychopathology. Submitted for publication.

Rescorla R. A. (1978) Some implications of a cognitive perspective on Pavlovian conditioning. In Cognitive Processes in Animal Behavior (Edited by Hulse S. H., Fowler H. and Honig W. K.), pp. 15-50. Erlbaum, Hillsdale, N.J.

Seligman M. E. P. (1971) Phobias and preparedness. Behav. Ther. 2, 307-320.

Stampf T. G. and Levis D. J. (1967) Essentials of implosive therapy: a learning-theory-based psychodynamic behavioral therapy. J. abnorm. Psychol. 72, 496-503.

Wolpe J. (1958) Psychotherapy by Reciprocal Inhibition. Stanford Univ. Press, Stanford, Calif.

Wolpe J. (1982) The Practice of Behavior Therapy, 3rd edn. Pergamon Press, New York.

Wolpe J. and Rachman S. (1960) Psychoanalytic "evidence": a critique based on Freud's case of Little Hans. J. nerv. ment. Dis. 131, 135-148. 